\title{
INTERAÇÃO ENTRE OS AGENTES DA BIBLIO- TECA UNIVERSITÁRIA DA UFSC: APLICAÇÃO DO FRAMEWORK GC@BU
}

\author{
Interaction between the university library of agents UFSC: the Framework GC@BU application
}

\author{
Roberta Moraes de Bem (1), Joana Carla Felicio (2), Tatiana Rossi (3) \\ (1) Universidade Federal de Santa Catarina, Florianópolis, Santa Catarina, roberta.bem@ufsc.br (2) joa- \\ na.felicio@ufsc.br (3) tatiana.rossi@ufsc.br
}

\begin{abstract}
Resumo
A caracterização da Biblioteca Universitária como um Sistema Adaptativo Complexo (SAC) na concepção do Framework GC@BU inclui a identificação de quatro processos: Reconhecimento, Variação, Interação e Seleção. Neste artigo será apresentado os aspectos relativos ao levantamento dos padrões de interação entre os agentes da Biblioteca Universitária da UFSC (BU/UFSC). Por meio do trabalho da Comissão de Gestão do Conhecimento da BU/UFSC, foi possível identificar, avaliar e registrar os elementos de interação. Foram mapeadas as principais formas de interação entre os agentes, o que influencia tais interações e as intervenções que poderiam mudar os padrões de interação para a melhoria do sistema. Conclui-se que a partir desta experiência foi factível visualizar ações, propor e aplicar melhorias. Apesar de não ter sido uma tarefa simples, o resultado foi considerando bastante positivo, não apenas para se ter consciência da complexidade da organização BU/UFSC, mas sobretudo para apoiar mais consistentemente o processo de tomada de decisão.
\end{abstract}

Palavras-chave: Gestão do Conhecimento; Bibliotecas Universitárias; Sistemas Adaptativos Complexos; Interação.

\section{Introdução}

A Gestão do Conhecimento (GC) valoriza os ativos intangíveis da organização, propicia competitividade, melhoria organizacional e inovação. Dalkir (2011) aponta que a atividade de coordenação sistemática e deliberada de pessoas, tecnologia, processos e estrutura de uma organização a fim de agregar valor por meio da reutilização de conhecimentos e da inovação, é alcançada por meio da criação, compartilhamento, aplicação do conhecimento, além das lições aprendidas e melhores práticas que agregam valor quando na reutilização de conhecimentos, e proporcionam o fortalecimento da memória corporativa.

A biblioteca universitária é considerada fundamental na estrutura da universidade devido ao seu caráter de promover acesso e disseminação da informação, além da geração do conhecimento, especialmente quando passou a ter a função de servir, também, como repositório e disseminadora do conhecimento estreitando a ligação entre o conhecimento produzido pela comunidade aca-

\begin{abstract}
The characterization of the University Library as a Complex Adaptive System (CAS) in designing the Framework GC@BU includes identifying four processes: Recognition Variation, Interaction and Selection. This paper deals with those aspects of the survey of patterns of interaction between agents of the University Library of the UFSC (BU/UFSC). Through the work the Knowledge Management Commission of the BU/UFSC, it was possible to identify, assess and record the interaction elements. The main forms of interaction between agents, which influences such interactions and interventions that could change patterns of interaction for improving the system were mapped. In conclusion from this experience was possible to view actions, propose and implement improvements. Although it was not a simple task, the result was extremely valuable, not only to be aware of the complexity of the BU/UFSC organization, but above all to support more consistent decision-making process.
\end{abstract}

Keywords: Knowledge Management; University Libraries; Complex Adaptive Systems. Interaction.

dêmica e os que necessitam desse conhecimento (Castro, 2005).

A biblioteca universitária trabalha o conhecimento a todo o momento, por isso é importante acompanhar a evolução desta temática, considerando que a $\mathrm{GC}$, traz variados benefícios, tais como: compartilhamento de experiência entre os colaboradores, integração de informações, racionalização de tarefas, melhoria da comunicação, amparo aos processos decisórios; facilitação do compartilhamento de conhecimentos e troca de experiências com as demais bibliotecas (Dudziak, Villela e Gabriel, 2002).

Moresi (2001, p. 37) entende a GC como o conjunto de atividades que visa desenvolver e controlar todo tipo de conhecimento em uma organização, com o propósito de apoiar a tomada de decisão. Fazer GC implica em estabelecer "políticas, procedimentos e tecnologias que sejam capazes de coletar, distribuir e utilizar efetivamente o conhecimento, representando fator de mudança no comportamento organizacional". 
Nesta perspectiva corrobora-se com o entendimento de que a GC em bibliotecas universitárias precisa estar ancorada nas demais atividades da organização para que ocorra de forma natural e possa ser diagnosticada, implementada e avaliada (Bem, 2015).

Como ferramenta para trabalhar a GC nas bibliotecas universitárias, Bem (2015), propõe um framework construído para esta finalidade - Framework de Gestão do Conhecimento para Bibliotecas Universitárias (GC@BU). Tal proposta foi constituída por meio de análise da literatura e submetida para validação no $I$ Workshop Gestão do Conhecimento em Bibliotecas Universitárias (2014) ${ }^{(1)}$ evento realizado na UFSC que reuniu um grupo de gestores de bibliotecas universitárias do Brasil, com o objetivo de fomentar as discussões na área de Gestão do Conhecimento em Bibliotecas Universitárias, por meio de palestras e compartilhamento de experiências.

O Framework GC@BU está dividido em três módulos: a) Coordenação de Gestão do Conhecimento; b) Recursos de Conhecimento e; c) Espaços de Aprendizagem/Conhecimento. Tais elementos estão sobrepostos a um plano de fundo que representa o Sistema Adaptativo Complexo (SAC), além da Standard for Libraries in Higher Education da Association of College and Research Libraries (ACRL), conforme apresentado na Figura 1. A concepção de biblioteca universitária como um SAC significa compreendê-la como um organismo dinâmico, composto de interações e em constante modificação.

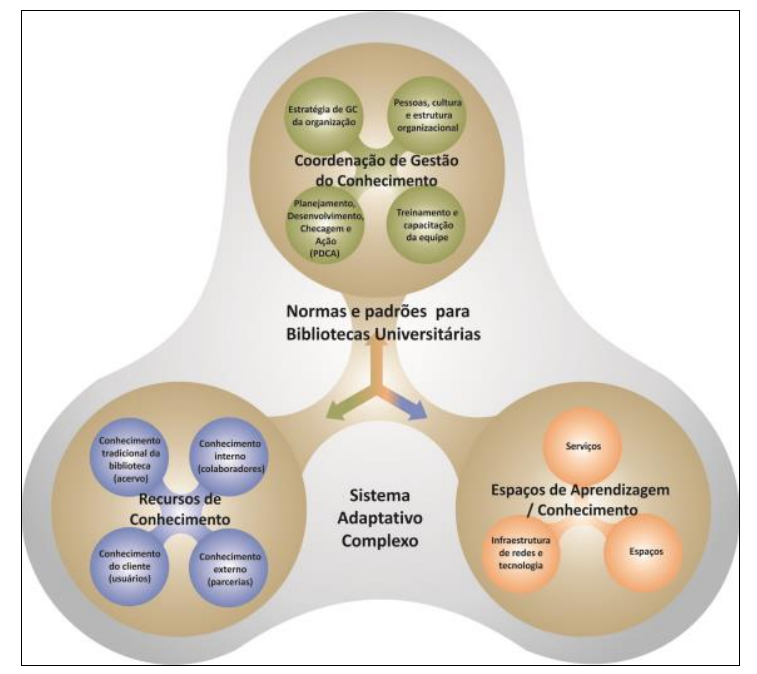

Figura 1. Framework de Gestão do Conhecimento para Bibliotecas Universitárias (GC@BU) (Bem 2015, p. 204).

Desta forma, para trabalhar a GC na Biblioteca Universitária da Universidade Federal de Santa Catarina (BU/UFSC), utilizou-se como base o Framework GC@BU, em trabalho colaborativo com a equipe.
A BU/UFSC é um órgão vinculado a Reitoria desta Universidade, constituído de uma direção administrativa e técnica centralizada, alocada na Biblioteca Central - BC, e de unidades setoriais distribuídas nos Centros de Ensino do Campus de Florianópolis (Biblioteca Setorial do Colégio Aplicação - BSCA; Biblioteca Setorial do Centro de Ciências Agrárias - BSCCA; Biblioteca Setorial do Centro de Ciências da Saúde - Medicina - BSCCS-M; Biblioteca Setorial do Centro de Ciências da Educação - BSCED; Biblioteca Setorial do Centro de Ciências Jurídicas - BSCCJ; Biblioteca Setorial do Centro de Ciências Físicas e Matemáticas BSCFM e Sala de Leitura José Saramago - SLJS), bem como em outros Campi da UFSC (Biblioteca Setorial do Campus de Araranguá - BSARA; Biblioteca Setorial do Campus de Blumenau - BSBLU; Biblioteca Setorial do Campus de Curitibanos - BSCUR; Biblioteca Setorial do Campus de Joinville - BSJOI) (Universidade Federal de Santa Catarina, 2015b).

A etapa inicial da implementação do Framework GC@BU consiste em entender a BU/UFSC como um SAC. Tal Sistema é constituído de alguns processos que são essenciais para este entendimento: a) reconhecimento; b) variação; c) interação; d) seleção, conforme apresentado na Figura 2.

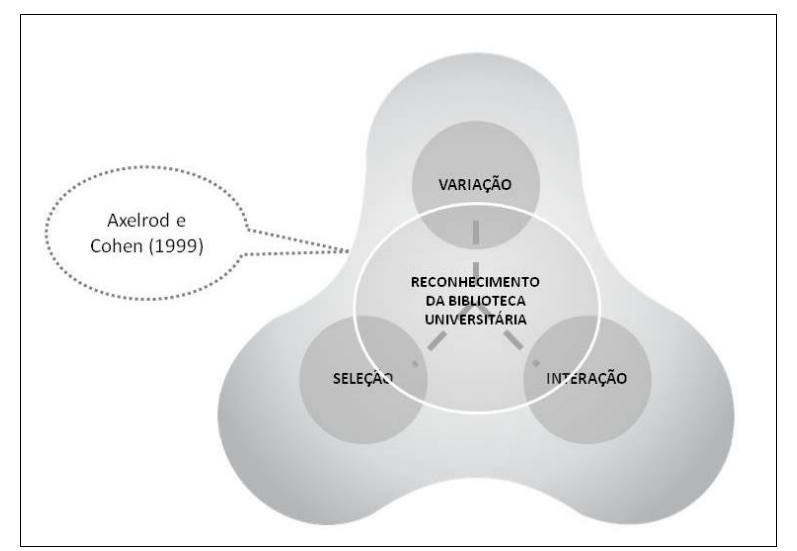

Figura 2. Biblioteca Universitária como Sistema Adaptativo Complexo (Bem 2015, p. 206).

O "Reconhecimento" da BU/UFSC como um SAC (2) foi "um exercício de identificar, avaliar e registrar os elementos fundamentais que constituem a biblioteca, tal como responder as questões: Quem somos? Onde estamos? O que fazemos? Como fazemos? Por que fazemos? Para quem fazemos?" (Bem, et al., 2016). Nesta etapa foram identificados os agentes que constituem a BU/UFSC, os espaços de atuação, as estratégias e artefatos que são utilizados ao agir, bem como os processos desenvolvidos.

Dando continuidade ao trabalho de implementação do GC@BU na BU/UFSC, o presente artigo visa apresentar os resultados das discussões suscitadas no grupo de trabalho que integra a Comissão de Gestão do Conhecimento da BU/UFSC, pautadas nos critérios de verifi- 
cação constantes do Framework GC@BU, no que concerne a "Interação entre os agentes".

\section{Interação}

Um SAC consiste em agentes interagindo uns com os outros, seguindo padrões que podem ser chamados de regras, de modo a melhorar seu comportamento e consequentemente do Sistema em que está inserido, o que significa que os agentes interagem buscando uma forma de aprendizado (Stacey, 1996).

A interação caracteriza a vivacidade do SAC. Padrões de interação ajudam a determinar o que será bemsucedido para os agentes de um sistema, e isso, em contrapartida, ajuda na dinâmica dos padrões de interação entre eles. Sendo que o conhecimento destas ocorrências em cenários complexos pode auxiliar na previsão dos sistemas.

Se a complexidade está frequentemente enraizada nos padrões de interação entre os agentes, então podemos esperar que os sistemas apresentem dinâmicas cada vez mais complexas, quando ocorrem mudanças que intensificam a interação entre os seus elementos (Axelrod; Cohen, 1999).

Lao et al. (2008) consideram que a possibilidade de uma empresa prosperar está diretamente associada a sua capacidade de interação com outras organizações, além de sua adaptação ao ambiente de mudança. Nesta perspectiva que são trabalhados os elementos da interação como parte da caracterização da BU como um SAC.

Ademais, na era da "poligamia" em que em que as bibliotecas estão inseridas é necessário considerar, concomitantemente, acervos impressos e eletrônicos, de modo que a possibilidade de uma integração de recursos mais profunda decorre de uma abordagem, mais sistêmica em que o investimento em parcerias e relacionamentos é relevante para o desenvolvimento das bibliotecas (Neal, 2012).

Neste contexto, a interação no ambiente das bibliotecas é importante para a criação de serviços, para alavancagem do conhecimento, para a tomada de decisão, entre outros. Considerando que quando se tem a consciência das interações - como ocorrem, entre quais agentes, em que locais, quais elementos as influenciam -, o gestor obtém elementos que ajudam a manipular as interações para benefício do sistema.

\section{Procedimentos metodológicos}

O trabalho de implementação desta ferramenta na BU/UFSC teve início em maio de 2015, ocasião em que foi emitida a Portaria $n^{\circ} 849 / 2015 / G R$, de 25 de maio de 2015, nomeando uma "comissão permanente para implantar práticas e projetos de gestão do conhecimento apoiados no Framework GC@BU, com o ob- jetivo de melhorar o fluxo e mapeamento de informações e conhecimentos no Sistema de Bibliotecas da UFSC" (Universidade Federal de Santa Catarina, 2015a, p. 1).

O Framework GC@BU é composto de vários elementos, com critérios de verificação específicos. Esses elementos e seus respectivos critérios foram transcritos em planilhas, as quais norteiam o trabalho da Comissão. A comissão de GC reúne-se quinzenalmente para analisar os critérios de verificação do GC@BU e após as discussões registra uma avaliação (ruim, regular, bom) e elenca ações, com seus respectivos prazos e responsáveis, para a melhoria do referido indicador. Trata-se de um exercício de reflexão, discussão, avaliação e, por fim, de registro dos resultados.

A etapa, relatada neste artigo, consiste na análise do elemento "Interação" (Apêndice) da caracterização da biblioteca universitária como um SAC, que constitui a verificação das principais formas de interações entre os agentes, identificando com quem, como e onde os agentes interagem; o que influencia tais interações e; que tipo de intervenções é possível fazer para melhorar estas interações. A análise e discussão dos resultados deste mapeamento são apresentados nas seções a seguir.

\section{Análise e Discussão dos resultados}

As seções a seguir são nomeadas pelo argumento discutido em cada critério do Anexo A, considerando que apresentam o resultado das discussões ocorridas no grupo de trabalho que integra a Comissão de GC da BU/UFSC, pautadas nos "Critérios de verificação para identificação dos padrões de interação" (Apêndice) constantes do Framework GC@BU.

Para identificação das interações entre os agentes, antes é necessário conhecer quais são estes agentes. Tal atividade foi realizada anteriormente a apresentada neste artigo. Na definição dos critérios que compõem o reconhecimento da BU/UFSC como um SAC (Bem et al., 2016).

\subsection{Formas de interação entre os agentes}

O primeiro critério de verificação consiste em conhecer com quem os agentes interagem, isto é, estabelecer as relações entre os agentes, bem como identificar como e onde ocorrem estas interações. Ao refletir sobre este critério, constatou-se que a BU/UFSC não possui formalmente mapeadas as interações entre os agentes e, a partir deste exercício, foi possível elencar as principais formas de interações entre eles, como apresentado a seguir:

a) Agente usuário x Agente colaborador: as interações ocorrem por meio dos serviços oferecidos, na forma presencial ou a distância. $\mathrm{O}$ usuário interage com o colaborador no momento da entrada na 
biblioteca, por meio do serviço de controle do fluxo de entrada/saída de pessoas e acervos (catraca); no balcão de empréstimo; no serviço de atendimento ao usuário (serviços de coleções especiais, referência, circulação e periódicos). As interações ocorridas a distância são, em geral, por telefone ou email. Os serviços que mais incorrem nesta modalidade de interação são: o atendimento às demandas dos usuários de uma forma geral; os eventos promovidos; o empréstimo/devolução (nos casos dos alunos de Ensino a Distância);

b) Agente usuário x Agente colaborador bibliotecário: as interações ocorrem na forma presencial ou a distância, em geral através de telefone e e-mail. Em ambos os casos são interações em decorrência dos serviços que são realizados, podendo-se citar o serviço de atendimento ao usuário, que ocorrem especialmente nos setores chamados de "linha de frente" (referência/circulação/periódicos); o Programa de Capacitação, que tem por objetivo desenvolver as competências informacionais dos usuários, de forma a torná-los mais autônomos na busca e uso da informação e; os eventos que são promovidos pela biblioteca;

c) Agente colaborador x Agente colaborador: as interações ocorrem geralmente por e-mail, listas de discussão, reuniões e por meio de conversas informais do cotidiano. Há ainda projetos ou ações específicas que tem por objetivo favorecer a interação entre os colaboradores, é o caso do projeto "Bom dia Biblioteca" ou das happy hours, por exemplo. As chefias e os colaboradores bibliotecários envolvidos nos diversos grupos de trabalho, em geral são quem interagem mais frequentemente;

d) Agente fornecedor $\mathrm{x}$ Agente colaborador bibliotecário: as interações com os fornecedores, na maior parte das vezes, são realizadas pelo próprio colaborador bibliotecário, tendo em vista que geralmente estão relacionadas à aquisição de material bibliográfico/documental ou à infraestrutura (aquisição de mobiliário/equipamentos). Estas interações ocorrem normalmente de forma presencial, por meio das visitas dos representantes de fornecedores, e muito frequentemente por e-mail;

e) Agente parceiro professor x Agente colaborador bibliotecário: o professor interage mais diretamente com o bibliotecário, e, em geral, estas interações ocorrem por e-mail e presencialmente. A interação com o corpo docente é muito importante para que a biblioteca possa trabalhar em consonância com os três pilares de sustentação da Universidade (ensino, pesquisa, extensão). A BU/UFSC conta com a colaboração de professores em comissões/grupos de trabalho, além de desenvolver diversos projetos/eventos em parceria, tais como: Projeto Cinema Mundo; Seminário de Acessibilidade na Universidade; Círculo de Leitura; Livro de Contos da BU; Ciclo de debates periódicos UFSC; Ciclo de
Palestras; Oficina de Escrita Criativa, Programa de Capacitação; dentre outros;

f) Agente parceiro UFSC x Agente colaborador bibliotecário: as interações ocorrem, em geral, por e-mail ou de forma presencial por meio de reuniões. Tais interações consistem em colaboração entre os setores da UFSC para que a BU possa desenvolver suas atividades com êxito e promover serviços de qualidade. Isto porque, a biblioteca não pode e não deve trabalhar isoladamente, ela é parte de um todo que é a Universidade e as parcerias entre os setores são fundamentais. Como parceiros da BU, internos à UFSC, destacam-se: Departamento de Ciência da Informação (CIN); Departamento de Engenharia e Gestão do Conhecimento (EGC); Superintendência de Governança Eletrônica e Tecnologia de Comunicação e Informação (SETIC); Agência de Comunicação da UFSC (AGECOM); Laboratório de Novas Tecnologias (LANTEC); Laboratório de Educação a Distância (LED); Curso de Graduação de Cinema;

g) Agente parceiro Não UFSC x Agente colaborador bibliotecário: os "parceiros Não UFSC" são aquelas instituições externas à UFSC que mantêm algum vínculo com a BU, criando possibilidades de interação. São alguns "parceiros Não UFSC": Comissão Brasileira de Bibliotecas Universitárias (CBBU); Instituto Brasileiro de Informação em Ciência e Tecnologia (IBICT); Coordenação de Aperfeiçoamento de Pessoal de Nível Superior (CA$\mathrm{PES})$; International Association of University Libraries (IATUL); International Federation of Library Associations (IFLA); dentre outros. As interações entre estes agentes ocorrem geralmente por e-mail e na forma presencial, especialmente por meio de eventos e/ou comissões/grupos de trabalho; h) Agente usuário x Agente usuário: as principais interações ocorrem no espaço físico que a biblioteca disponibiliza e por intermédio dos projetos/serviços promovidos pela biblioteca que favorecem esta interação. Como por exemplo, pode-se citar o projeto "Café com Tato", promovido pelo Setor Acessibilidade Informacional com a finalidade de propiciar momentos de interação entre os seus usuários; assim como o "Projeto Cinema Mundo", que oportuniza o debate e a interação destes agentes por meio da projeção audiovisual. Além disso, percebe-se que as redes sociais são frequentemente utilizadas por estes agentes e se constituem em um espaço nato de interação.

\subsection{Fatores que influenciam as interações}

O segundo critério está relacionado à identificação dos elementos que influenciam as interações elencadas. Os resultados das discussões estão apresentados em formato de quadro (Quadro I), para facilitar a identificação da ocorrência dos mesmos elementos em interações 
diferentes. Pode-se perceber que quase a totalidade das interações identificadas são influenciadas pela necessidade de informação e/ou interesse dos agentes. Outro fator que, no entendimento da Comissão, também se constitui decisivo para o estabelecimento de interações é a proximidade física.

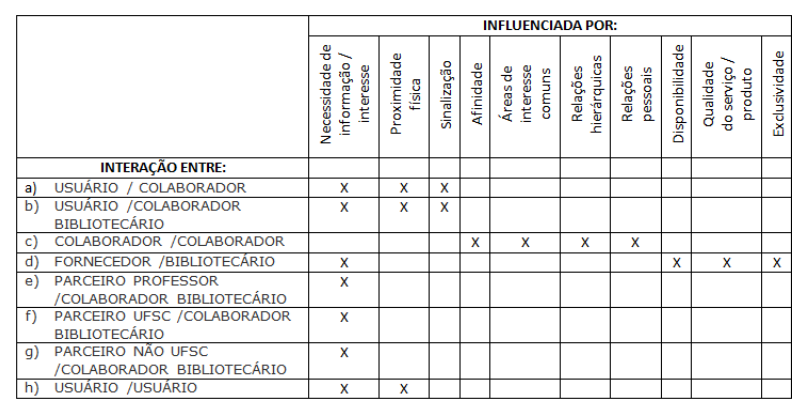

Quadro I. Elementos que influenciam as interações entre os agentes (Bem at al 2015).

Após mapear as formas de interações e quais aspectos as influenciam, a Comissão sentiu necessidade de registrar estas informações de forma mais visual, e a partir do desenvolvimento da Figura 3, foi possível pensar em quais ações poderiam ser propostas.

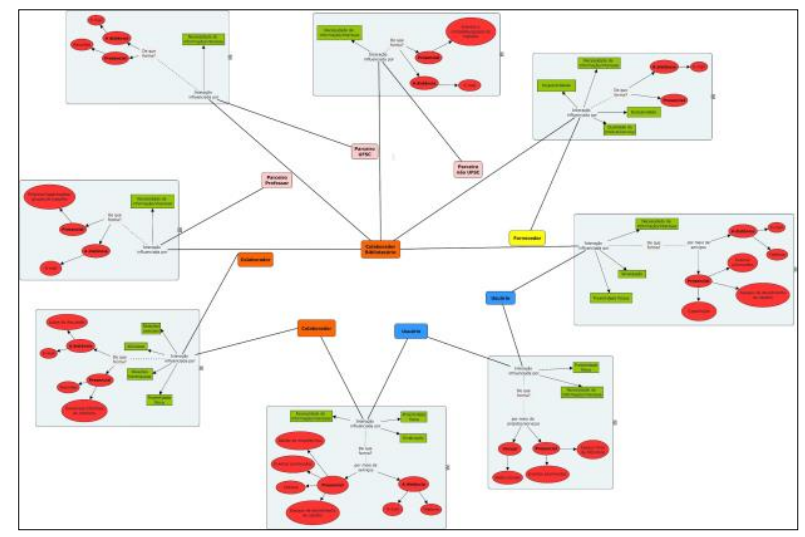

Figura 3. Mapa mental das interações da Biblioteca Universitária (Comissão de GC da BU/UFSC. Implementação do Framework GC@BU, 2015).

4.3 Identificação das intervenções que podem mudar os padrões de interação

No terceiro critério de verificação, foram analisadas as mudanças que poderiam ser realizadas de forma a serem proveitosas para o sistema como um todo. Neste sentido, a Comissão fez algumas sugestões de intervenções em cada uma das interações levantadas:

a) Agente usuário x Agente colaborador: melhorar a comunicação com o usuário, especialmente no que se refere à sinalização do espaço e dos acervos das bibliotecas;

b) Agente usuário x Agente colaborador bibliotecário: criar estações de informação com a finalidade de filtrar as demandas que podem ser resolvidas por outros colaboradores, encaminhando para os bibliotecários os usuários com demandas mais específicas; estabelecer barreiras físicas nos ambientes, para possibilitar o desenvolvimento de atividades que requerem maior nível de concentração;

c) Agente colaborador $\mathrm{x}$ Agente colaborador: resgatar projetos de GC (Bom dia Biblioteca); implantar/formalizar novas ações que promovam a interação entre os colaboradores de forma periódica. Ex. sistema de rodízios de função e estações de trabalho; boletins informativos; reuniões segmentadas; regulamentar a elaboração de atas de todas as reuniões e instituir meios de divulgação das mesmas para acesso e conhecimento de todos os colaboradores, tais como: intranet, pastas compartilhadas em rede, mailing, mural físico, arquivamento físico;

d) Agente fornecedor $\mathrm{x}$ Agente bibliotecário: propiciar apoio técnico ao bibliotecário nas funções burocráticas/administrativas, de forma que ele possa ter mais tempo para desenvolver atividades relacionadas ao fazer bibliotecário no que se refere a melhoria da coleção e infraestrutura; estabelecer diretrizes para as relações com os fornecedores baseadas na impessoalidade, moralidade, legalidade, publicidade, consistência, e demais princípios da administração pública; resgatar a estratégia de marketing da BU que consiste na disponibilização de um bibliotecário por Centro de Ensino para trabalhar de forma mais específica a divulgação dos recursos informacionais, bem como a capacitação da comunidade para o uso destes recursos. Tal estratégia visa apoiar a tomada de decisão nas atividades inerentes ao processo de desenvolvimento da coleção, especialmente no que se refere aos recursos eletrônicos, bem como identificar demandas específicas da comunidade. Conhecer as necessidades da comunidade universitária torna a relação entre BU e fornecedor mais consistente;

e) Agente parceiro professor x Agente colaborador bibliotecário: resgatar a estratégia de marketing da BU (um bibliotecário por Centro de Ensino), visando o estreitamento da relação com os alunos e professores, o levantamento de necessidades específicas, a divulgação do acervo e serviços da biblioteca; fornecer apoio aos grupos de pesquisa; institucionalizar a participação da biblioteca nos currículos dos cursos de graduação e pós-graduação, tornando obrigatória a visita orientada na biblioteca em todos os cursos de graduação e a participação dos alunos nos módulos de capacitação referentes a iniciação à pesquisa e a normalização dos trabalhos acadêmicos;

f) Agente parceiro UFSC x Agente colaborador bibliotecário: criar estratégias de aproximação com os setores, tais como: eventos, rodas de conversa, projetos, parcerias;

g) Agente parceiro Não UFSC x Agente colaborador bibliotecário: ampliar a representação da 
BU/UFSC junto às instituições, bem como reforçar a participação destas instituições nas ações promovidas pela biblioteca; criar uma comissão de comunicação e marketing para reforçar estas parcerias, criar estratégias - em conjunto com a direção da BU/UFSC - buscando ampliar estas relações. Tal comissão poderia ter como atribuições a formalização do marketing interno (um bibliotecário por Centro de Ensino); a gestão e realização de eventos; a definição de diretrizes para os meios de comunicação (Portal BU, redes sociais, BU Informa); dentre outras;

h) Agente usuário x Agente usuário: ampliar os espaços, físicos e virtuais, de interação entre os agentes, como exemplo: habilitar as funcionalidades colaborativas do Sistema Pergamum (catálogo automatizado da BU/UFSC); promover exposições interativas; disponibilizar salas para estudo em grupos; dentre outros.

\section{Conclusões}

Ao levantar os aspectos associados à interação entre os agentes da BU, como parte da caracterização da BU/UFSC como um SAC, foi possível, especialmente após representar os citados elementos por meio de um mapa mental, a possibilidade imediata de visualizar ações e propor melhorias, acompanhando a dinâmica que a vivacidade de um SAC sugere, especialmente em virtude de suas interações.

O exercício de pensar conjuntamente a organização em que se está inserido e sistematizar as ideias provenientes desta reflexão, não é uma tarefa simples. Entretanto, a experiência na BU/UFSC mostrou que o resultado deste esforço é extremamente válido para se ter consciência da complexidade da organização e apoiar mais consistentemente o processo de tomada de decisão.

Ao considerar as ações de melhoria propostas, na seção 4.3 (fluxo de informação, normalização de procedimentos, compartilhamento de conhecimento, mudanças estruturais, reaproveitamento de recursos), verifica-se a característica prática da ferramenta GC@BU, sendo que as ações sugeridas são de fácil implementação e podem gerar benefícios significativos em variados aspectos.

A percepção dos resultados na implementação de um projeto de GC é fundamental para a manutenção da atividade, tendo em vista que o trabalho de GC tem características pouco tangíveis, e que em geral desmotivam as equipes para a sua implementação, quando não se vislumbrar benefícios em curto prazo.

\section{Notes}

(1) Disponível em:

http://workshopgestaoconhecimentobu.paginas.ufsc.br.
(2) Os resultados da aplicação do Framework GC@BU no que se refere aos aspectos de "Reconhecimento da Biblioteca Universitária" foram apresentados em artigo aprovado Revista Ibero-Americana de Ciência da Informação (RICI), disponível em:

$<$ http://periodicos.unb.br/index.php/RICI/article/view/1 6129/12853>.

\section{Referências}

Axelrod, R. M.; Cohen, M.D. (1999). Harnessing Complexity: Organizational Implications of a Scientific Frontier. The Free Press, New York, NY, 1999.

Bem, R. M. de. (2015). Framework de gestão do conhecimento para bibliotecas universitárias. http://tede.ufsc.br/teses/PEGC0364-T.pdf (2016-01-05)

Bem, R. M. de et al. (2016) . Reconhecimento da biblioteca universitária como um Sistema Adaptativo Complexo (SAC): aplicação do framework GC@BU na Biblioteca Universitária da UFSC. // Revista Ibero-Americana de Ciência da Informação, 9:1 (2016) 87-107.

http://periodicos.unb.br/index.php/RICI/article/view/16129 (2016-07-18)

Castro, G.de. (2005). Gestão do conhecimento em bibliotecas universitárias: um instrumento de diagnóstico. 2005. http://pgcin.paginas.ufsc.br/files/2010/10/CASTROGardenia.pdf (2013-07-24).

Dalkir, Z. (2011). Knowledge Management in Theory and Practice, 2nd ed. MIT Press, Cambridge. 2011.

Dudziak, E. A.; Villela, M. C. O.; Gabriel, M. A. (2002). Gestão do conhecimento em bibliotecas universitárias // Seminário Nacional de Bibliotecas Universitárias, Recife. 12 (2002) 1-12.

Lao, G. et al. (2008). Research on Organizational Knowledge Sharing Framework based on CAS Theory. // International Conference on Service Systems and Service Management, Melbourne, 5, 2008. http://dx.doi.org/10.1109/ICSSSM.2008.4598540 (2016-11-13).

Moresi, E. A. D. (2001). Inteligência organizacional: um referencial integrado, Ciência da Informação, 30: 2. (2001). 35-46. http://www.scielo.br/pdf/ci/v30n2/6210.pdf. (2016-11-11).

Neal, J. G. (2012). Opportunities for systematic change in the academic research library: elements of the post-digital library. // Insights, 25: 1. (2012). 92-97. http://dx.doi.org/10.1629/20487754.25.1.92 (2016-11-11).

Stacey, R. (1996). Emerging Strategies for a Chaotic Environment, Long Range Planning, 29: 2 (1996) 182-189. http://dx.doi.org.ez46.periodicos.capes.gov.br/10.1016/0024 6301(96)00006-4 (2014-04-02).

Universidade Federal de Santa Catarina (2015a). Portaria $\mathrm{n}^{\circ}$ $849 / 2015 / G R$, de 25 de maio de 2015. Florianópolis.

Universidade Federal de Santa Catarina (2015b). Sistema de Bibliotecas Universitárias. Florianópolis, 2015b. http://portal.bu.ufsc.br/ (2016-08-14).

Copyright: (C) 2017, Bem et al. This is an open-access article distributed under the terms of the Creative Commons CC Attribution-ShareAlike (CC BY-SA), which permits use, distribution, and reproduction in any medium, under the identical terms, and provided the original author and source are credited.

Received: 2015-11-13. Revised:2016-07-26 Accepted: 2016-12-20 


\begin{tabular}{|c|c|c|c|c|}
\hline $\begin{array}{l}\text { CRITÉRIOS DE VERIFICAÇÃo } \\
\text { (A Biblioteca Univer sitária...) } \\
\end{array}$ & $\begin{array}{l}\text { Relação } \\
\text { com... }\end{array}$ & Nível & Prazo & Resp. \\
\hline $\begin{array}{l}\text { 1. Tem mapeadas as principais formas de } \\
\text { inter acões entre os agentes (Como? Onde? Com } \\
\text { quem?). }\end{array}$ & $\begin{array}{l}\text { Pessoas, } \\
\text { cultura } \\
\text { e estru- } \\
\text { tura }\end{array}$ & & & \\
\hline $\begin{array}{l}\text { 2. Sabe o que influencia tais interações. } \\
\text { Exemplos: relações hierárquicas, vínculos de } \\
\text { amizade, proximidadefísica. }\end{array}$ & & & & \\
\hline $\begin{array}{l}\text { 3. Identifica que intervencões poderiam mudar } \\
\text { os padrões de interação (de forma que } \\
\text { pudessem ser proveitosas para o sistema como } \\
\text { um todo). Conhecer possíveis vizinhanças fisicas } \\
\text { ou conceituais de interação que necessitam } \\
\text { auxílio para se formar, ou que mereçam ser } \\
\text { inter rompidas. Exemplos: } \\
\checkmark \quad \text { Estabelecimento de barre iras físicas } \\
\text { (biombos) para quebrar o fluxo de } \\
\text { interações; } \\
\text { Criação de comuni dades de prática (espaço } \\
\text { conceitual) para proporcionar a interação } \\
\text { entre determinados agentes; } \\
\text { Sistemas de rodízio de função e estação de } \\
\text { trabalho em determinado dia da semana } \\
\text { ou mês, para que os colegas possam } \\
\text { perceber de que forma suas atividades } \\
\text { impactamnotrabalho dosdemais. }\end{array}$ & $\begin{array}{l}\text { Conheci } \\
\text {-mento } \\
\text { Interno }\end{array}$ & & & \\
\hline
\end{tabular}

Critérios de verificação para identificação dos padrões de interação Fonte: Bem (2015, p. 214-215). 